\title{
Equipos interdisciplinarios: Nuevas formas de prestar servicios de salud, nuevas oportunidades para el Trabajo Social
}

\author{
Interdisciplinary teams: New ways of delivering health \\ services, new opportunities for Social Work
}

\begin{abstract}
Mg.C Karla GonZÁlez
Karla González es Asistente Social de la Universidad Católica de Chile, y candidata a magíster en Trabajo Social en la misma Universidad. Actualmente trabaja como Asistente Social en el Centro de Salud Familiar Juan Pablo II, proyecto de los Centros de Salud Familiares Áncora dependiente de la Universidad Católica, cuya función universitaria se expresa en docencia, investigación y desarrollo de innovaciones, orientada hacia el modelo de atención en salud familiar. Su dirección postal es Avenida La primavera 02870, Paradero 31 Santa Rosa, La Pintana y su dirección e-mail es: kgonzalezsuitt@gmail.com
\end{abstract}

\begin{abstract}
Resumen
En este documento se exponen algunas dimensiones claves sobre interdisciplinariedad, identificados en una investigación que estudió las reuniones de equipos interdisciplinarios de salud familiar en uno de los Centros de Salud Familiar del Proyecto Áncora UC, a fines del año 2004. En esta investigación se indagó en los contenidos de las reuniones de equipo y en la interacción de las distintas disciplinas que participan en éstas. Su objetivo general fue "Conocer, comprender y analizar cómo se está desarrollando y conjugando el Modelo Biopsicosocial con el enfoque interdisciplinario al interior de los equipos de salud, a través del análisis de sus reuniones". El objetivo de este artículo es dar cuenta los resultados de dicha investigación referentes al trabajo interdisciplinario de los equipos de salud familiar y con ello hacer una reflexión en torno a las implicancias que el tema sugiere para la formación en Trabajo Social.

Palabras claves. Interdisciplina, equipos de salud, formación en Trabajo Social.

Abstract

This study analyzes some key dimensions about interdisciplinary work identified through the content analysis of interdisciplinary meetings, these meetings were developed in one of the Project Áncora's family health center. The study was developed at the end of year 2004. The study's general aim was "To know, to understand and to analyze how it is developed and brought together the Biopsicosocial Model with the interdisciplinary approach into the teams of health, across the analysis of their meetings". The objective of this article is to present the results of the above mentioned research related to family health teams' interdisciplinary work and from there, to develop some implications for training in social work.
\end{abstract}

Key words. Interdiscipline, teams of health, training in social work.

\section{Introducción}

Existen diversas áreas de reflexión sobre la formación en la profesión de Trabajo Social: respecto del currículum y los contenidos de éste, las prácticas como parte de la formación, la relevancia de la investigación y la esencial articulación dialéctica entre la teoría y la práctica, entre muchos otros temas. Claramente, la profesión ha visto transformaciones en todos aquellos temas, desde su origen hasta hoy, pudiendo apreciarse diversas posturas en torno a cómo se debe formar Trabajadores
Sociales, qué deben aprender, qué deben hacer, en qué momento deben realizar prácticas profesionales, cuáles son los ámbitos a los que deben concurrir y, finalmente en la actualidad, cómo se articulará la teoría y la práctica una vez egresados. Se ha escrito sobre los fenómenos sociales en torno a los cuales actúan las diversas profesiones, Trabajo Social es sin duda una ocupación que se ubica en distintos contextos sociales y el ejercicio profesional se desarrolla desde cada ámbito en relación a otras profesiones apuntando a la comprensión 
compleja de los fenómenos.

Por ello, es relevante un análisis que traspase la particularidad de diferentes disciplinas cuya incumbencia podría ser específica, la comprensión de las situaciones que se abordan merecen un análisis complejo que ponga en juego las especificidades de los saberes profesionales construyéndose a través de un diálogo fecundo y horizontal, los objetivos de intervención, que den paso a nuevas estrategias de acción e intervenciones particulares que se configuren desde la complementariedad. Desde este punto de vista, no habría una disciplina particular que interviene en lo social, sino una "mirada disciplinar que se hace cargo de ciertos aspectos de una situación que emerge de lo social" (Cazzaniga, 2007, p. 107).

El sector salud es un área social específica donde interactúan indudablemente ocupaciones cuyo origen se ubica en variadas disciplinas, dentro de las cuales Trabajo Social en Chile es tradicionalmente incluido $^{1}$ (Goyeneche, 1927, p. 150-151. En Aylwin et. al., 2004, p. 66-70). Inicialmente, el área de intervención se abocó en gran medida a la educación de la población más necesitada de la sociedad respecto de situaciones de emergencia, como aquellas condiciones de higiene y salud que correspondían a la generación de graves enfermedades infecciosas como la TBC, y enfermedades venéreas, entre otras, trabajando junto a profesionales médicos en la consecución del objetivo de mejorar las condiciones ambientales y sociales para el logro de intervenciones eficaces. Posteriormente, en consecuencia con la transición epidemiológica de Chile y sin duda, con el desarrollo de otras profesiones como la psicología, sociología o terapia ocupacional, el rol de la profesión en el ámbito de la salud ha ido adquiriendo tareas más específicas (Aylwin et. al. 2004, p. 130-140).

En este contexto, el concepto de salud ha evolucionado, pasando desde una comprensión como mera ausencia de enfermedades, hacia un estado de completo bienestar físico, psicológico y social (OMS, 2009) que configura lo que se ha denominado Enfoque Biopsicosocial de Salud. Esto a su vez, implica la transición desde un modelo -arraigado en los equipos de salud- basado en un paradigma que conceptúa salud y enfermedad desde una perspectiva biomédica, hacia otro que ofrezca una perspectiva más amplia y apropiada, para enfrentar los nuevos desafíos en salud.

La Facultad de Medicina y en especial el Departamento de Medicina Familiar de la Universidad Católica de Chile, llevaron a cabo un proyecto concreto en relación a la atención primaria y la ejecución del modelo de Salud Familiar que materializa el enfoque biopsicosocial de comprender la salud. Nacen así los Centros de Salud Familiar (CESFAM) Áncora UC, que siendo parte de la Red de Salud UC, prestan un servicio de atención primaria en sectores poblacionales vulnerables del sector Sur Oriente de la Región Metropolitana ${ }^{2}$. El propósito de Áncora es la implementación de un modelo de atención primaria de alta calidad y eficiencia (...) que sirva para el desarrollo de políticas públicas en el ámbito de la Atención Primaria de Salud chilena (Áncora-UC, 2009).

El $1^{\circ}$ de abril del año 2004, se abren las puertas del CESFAM Madre Teresa de Calcuta, el cual fue el primero de los centros de atención primaria que incluye la Red de Salud Áncora UC, donde el Equipo de salud interdisciplinario es uno de los pilares fundamentales para implementar el enfoque biopsicosocial, dado que los profesionales que participan son los llamados a hacer efectivos los cambios de enfoque propuestos por la autoridad sanitaria. Entre los miembros del equipo de salud se cuentan: médicos, enfermeros, psicólogos, trabajadores sociales, odontólogos, matrones, y otros.

Varios autores han conceptualizado el término interdisciplinario, habiendo en general acuerdo sobre las diferencias de éste con otras expresiones similares tales como lo monodisciplinario, multidisciplinario o transdisciplinario. Martínez (2003) habla de un continuo en donde cada uno de estos conceptos corresponde a un nivel -monodisciplinario, multidisciplinario, interdisciplinario y transdisciplinario- agregando que el énfasis tiene lugar en la naturaleza de la integración que se hace tanto del proceso investigativo como de los resultados o ha-

1 Cabe mencionar que la primera Escuela de Visitadoras Sociales en Chile fue promovida y fundada en 1925 por un médico, preparando inicialmente Visitadoras Sociales que trabajaban en hospitales, y cuyas tareas consistían en colaborar con el médico en la prevención y cura de enfermedades; evaluar el contexto personal, familiar y socio cultural de los asistidos; realizar diagnóstico social del enfermo y su familia a fin de apoyar en el logro de las indicaciones médicas que se le hayan otorgado; velar por el bienestar de los niños abandonados.

2 Actualmente, se encuentran operativos tres Centros de Salud Familiar en las comunas de Puente Alto y La Pintana que configuran una red de atención. Estos centros se constituyen además en un campo de formación e investigación para alumnos y profesionales de diversas carreras dictadas en la Universidad Católica, entre ellas, medicina, enfermería, psicología, Trabajo Social, kinesiología, entre otros. 
llazgos de las diferentes disciplinas. Implícitos en el concepto de "integración" se encuentran los conceptos de "comprensión" y de "extensión" del proceso y del análisis. Conviene por ello establecer al menos la diferenciación conceptual entre estas expresiones.

Lo monodisciplinario, de acuerdo a Martínez (2003, p. 114) se orienta a un trabajo dentro del ámbito de una sola disciplina, dando prioridad a la extensión por sobre la comprehensión. Su versión extrema es la hiperespecialización. Por ello, es considerado un enfoque reduccionista e incapaz de abordar las complejidades del mundo actual.

Por multidisciplinario se ha entendido una yuxtaposición o agregación de disciplinas. Como lo señala Klein (1990, p. 56), "Es esencialmente aditivo, no integrativo (...) su relación puede ser mutual y acumulativa, pero no interactiva". Martínez (2003, p. 115) agrega que cada disciplina es independiente en su trabajo sin existir necesidad o interés por conocer el trabajo de las otras. En este sentido, Gyarmati (1991) argumenta que este estilo de trabajo permite organizar diversos estudios en torno a un problema en común, donde cada uno representa el enfoque particular de cada disciplina y los principios, métodos y conceptos principales de cualquier profesión no se modifican por la influencia de los principios de otra.

Por su parte, lo interdisciplinario implicaría integración y síntesis, siendo la finalidad lograr que los elementos que constituyen dos o más disciplinas se integren entre sí, orientándose a las bases del conocimiento y contribuyendo a que cada una de las disciplinas implicadas se perfeccione. (Gyarmatti, 1991). Klein (1990, p. 63) complementa el significado de este concepto -apoyándose en diversos autores- integrando la idea de una asimilación recíproca entre las disciplinas participantes, como un elemento fundamental para la interacción, siendo de especial aporte para su desarrollo el trabajo en equipo. Finalmente, Martínez (2003, p. 116) aporta que en una investigación interdisciplinaria "la coordinación, la comunicación, el diálogo y el intercambio son esenciales, para traducir los términos propios, aclarar los lenguajes ambiguos, seguir, aunque sea parcialmente, procedimientos metodológicos similares, y, en general, tratar de compartir algunos de los presupuestos, puntos de vista y lenguajes de los otros".

Un método de trabajo que trasciende a los anteriores es el transdisciplinario, que ha sido conceptualiza- do más recientemente como aquel que permite una completa integración teórica y práctica, dando lugar a un nuevo mapa cognitivo sobre el problema en cuestión (Martínez, 2003, p. 117).

Indudablemente, cada método es útil en distintos contextos, de acuerdo a los objetivos del equipo y de las investigaciones o acciones que se deseen emprender. Sin embargo, en el caso especifico de un equipo perteneciente a un centro de atención primaria de salud y considerando los desafíos de la implementación del Modelo de Salud Familiar, se desprende que las principales oportunidades que brinda el nuevo paradigma biopsicosocial no reside sólo en la incorporación de equipos de trabajo con integrantes de diversas disciplinas (equipo multididsciplinario) que se dedican a atender a determinada población en aras de facilitar la figura "médico de cabecera", sino también en la posibilidad de integrar los conocimientos que cada una de aquéllas puede aportar (equipo interdisciplinario) en pro de una mejora en la calidad del servicio otorgado a los usuarios, comprendiendo que estos últimos se desenvuelven en un contexto familiar, perteneciendo a una comunidad y cultura particular.

Uno de los desafíos que emergen en este sentido es compatibilizar las lógicas de cada una de las disciplinas, en especial las provenientes del área biomédica con un modelo de atención de salud que involucre disciplinas de otras ramas científicas distintas, precisamente porque biomédica se caracteriza por inculcar que el médico tiene la potestad de un individuo enfermo (Tobeña, 2006, p 11).

Frente a lo anterior, los CESFAM Áncora UC han implementado como estrategia de los equipos de salud las reuniones de equipo, realizándose una vez por semana y prolongándose por dos horas. Esta es una de las formas de trabajo, a través de la cual se pretende llevar a la práctica el trabajo en equipo interdisciplinario y con enfoque biopsicosocial que se propone en el Modelo de Atención con Enfoque Familiar.

Es aquí donde también surge la posibilidad de reflexionar sobre la formación y educación en Trabajo Social y preguntarse por la vinculación que ambas podrían tener con el modelo interdisciplinario que se describe anteriormente. En las páginas siguientes, se dará cuenta de algunos elementos analizados a la luz del estudio de las reuniones de equipos interdisciplinarios ${ }^{3}$ de salud familiar en un Centro

3 En las cuales participan médicos, enfermeras, psicólogos, trabajadores sociales, odontólogos, auxiliares paramédicos, auxiliares dentales, administrativos, becados de medicina familiar, internos de medicina, internos de enfermería, alumnos de Trabajo Social, entre otros. 
de Salud Familiar de la Red de Salud Áncora UC, en el cual -entre otros aspectos- se profundizó en la interacción de los miembros de los equipos y la puesta en práctica de la interdisciplinariedad en las reuniones.

Posteriormente, la discusión se centrará en cómo entra en este ámbito la noción de interdisciplina y qué posibilidades se observan a en torno a la formación de trabajadores sociales.

\section{Metodología}

El proceso de investigación desarrollado, se llevó a cabo a través de una metodología cualitativa, que permitió captar valoraciones, actitudes, conductas y motivaciones, abordando los dinamismos de la realidad particular. El objeto de estudio lo constituyeron los equipos de atención de salud Azul y Verde del CESFAM Madre Teresa de Calcuta, perteneciente a la Red de Salud Áncora UC. La escala del estudio es micro-social, dado que se centró en el contexto específico de dichos equipos de salud, colocando énfasis en los procesos internos que cada uno de éstos llevan a cabo. El nivel de profundidad fue descriptivo, puesto que se buscaba responder principalmente a las preguntas ¿Qué?, ¿Cómo?, ¿Cómo se relacionan sus partes?, entre otras. El análisis se realizó a partir de fuentes primarias como registros escritos de reuniones de equipo y entrevistas en profundidad.

El registro consistió en grabaciones de audio de cuatro reuniones semanales de cada equipo asistencial de salud, las cuales fueron transcritas en textos para, posteriormente, ser analizados. Luego de esto se desarrollaron nueve entrevistas en profundidad a diversos profesionales y no profesionales de ambos equipos entre los que se cuentan: una recepcionista, una paramédico, dos asistentes dentales, una matrona, dos asistentes sociales y dos médicos familiares. Entre estos cuatro últimos se incluyó a las dos jefas de equipo. La decisión por cada uno de los entrevistados fue con el objeto de captar las percepciones de aquellos estamentos ocupacionales que participaban menos en las reuniones de equipo (administrativos, paramédicos y matronas), a médicos familiares como referentes de la salud familiar y a trabajadoras sociales como referentes de la profesión en la que se enmarca la investigación.

Como técnica de análisis de los datos, se utilizó el método de análisis de contenido, orientado a analizar y estudiar en detalle el contenido de una comunicación escrita, oral o visual (Pérez, 1994, p. 102). Este método comprende un conjunto de técnicas de análisis de comunicaciones tendentes a obtener indicadores por procedimientos sistemáticos y objetivos de descripción del contenido de los mensajes, permitiendo la inferencia de conocimientos relativos a las condiciones de producción/recepción (variables inferidas) de estos mensajes (Bardín, 1996, p. 32).

\section{Presentación de resultados}

"El problema de acceder y fundamentar el pluralismo sin renunciar a la idea de unidad, radica en comprender que la propia noción de unidad contiene como requisito funcional una matriz de distinciones. Lo anterior posibilita no renunciar sino resignificar un concepto de totalidad y diferenciarla de los mecanismos metafísicos de la totalización" (Jay, 1984 en Matus, 2005, p. 51).

Del análisis de las reuniones en el contexto de salud, y a fin de indagar qué método de trabajo se usa a través de las conversaciones registradas, se desprende una serie de contenidos referidos a la particularidad de interacción entre diversas ocupaciones en un equipo de salud familiar en atención primaria.

A la luz de los equipos interdisciplinarios, Klein (1990, p. 150) argumenta que el éxito de éstos no se debe sólo a coincidencias o encajes de personalidades, sino un tema que requiere duro trabajo y que permite a su vez identificar nuevas necesidades.

En relación a la interdisciplinariedad en sí misma, la autora sostiene que constituye un proceso integrativo que aunque no posee una progresión lineal, incluye algunos pasos, tales como (Klein, 1990, p. 188-189):

- Definir el problema, tema o pregunta,

- Determinar todos los conocimientos necesarios -incluyendo asignar a representantes disciplinarios y consultores,

- Desarrollo de un marco integrativo y preguntas de investigación apropiadas,

- Especificación de los estudios que serán asumidos,

- Reunir material actual y buscar nueva información,

- Resolver conflictos disciplinarios trabajando por un vocabulario común (enfocado al aprendizaje recíproco en equipo de trabajo),

- Construir y mantener la comunicación a través de técnicas integrativas,

- Recopilar todos los aportes conceptuales y evaluar si son adecuados y relevantes, 
- Ratificar las soluciones propuestas,

- Decidir sobre el manejo o disposición futura de la tarea, proyecto, paciente o currículum.

Asimismo, se identifican varias técnicas integrativas para desarrollar por los equipos de trabajo o por las personas, entre las cuales destacan las reuniones de equipos, presentaciones internas y externas, articulación continua de educación, articulación de diferencias entre los miembros de un equipo, entrenamiento en habilidades de interacción grupal, reuniones comunes de enseñanza, entre otros (Klein, 1990, p. 189-190).

Es importante en esta línea profundizar en las reuniones de equipo, al instaurarse como actividad periódica de los equipos de salud, y por otra parte, al ser un espacio en el cual comparten conversaciones, opiniones y diálogos las variadas disciplinas que forman parte del trabajo en el sector salud. Éste puede ser un lugar que permite y enseña a participar grupalmente, ayudando a democratizar las relaciones y discutir las diferencias, lo cual se vincula con el desarrollo de un equipo capaz de asumir desafíos y de participar creativamente en la solución de los problemas (Cavieres y Valdivieso, 2004, p. 33).

Temas en torno al funcionamiento interno del centro de salud, criterios de atención a los usuarios y trabajo con enfoque familiar fueron foco principal de las conversaciones de los equipos de salud. Como ejemplo de interacción de las diversas disciplinas en el equipo de salud, se considera importante destacar la estructura de la presentación de las familias, pues en cada una de ellas se identifican tres procesos interesantes que se producen naturalmente mientras se experimentan los diálogos: presentación, análisis y toma de decisiones.

En todos los temas existen protagonistas que acuden al CESFAM con algún síntoma, cada uno de ellos vive en un contexto familiar, social y cultural, información considerada por los equipos en las discusiones. Esto es quizás el valor agregado de la intervención de un Centro de Salud Familiar en una comunidad, pues las reflexiones que se levantan en las reuniones de equipo, son el reflejo de intervenciones que trascienden del modelo biomédico, profundizando en las relaciones que puedan existir entre la enfermedad y el contexto que contiene al sujeto.

\section{Presentación}

Indistintamente se presentan las familias en ambos equipos, por lo general, dibujando su genograma ${ }^{4}$ en una pizarra, donde también se detallan cuales son las características principales de los miembros del grupo familiar, sus actividades, su historia de salud, quienes se atienden en el CESFAM, etc. Asimismo, el o los profesionales que escogen presentar a una familia, solicitan información al equipo, respecto de si alguien más la conoce o ha trabajado con ésta. Con frecuencia se hace alusión al motivo de consulta del "paciente índice" o protagonista de la historia presentada, lo cual suele ser algún síntoma que afecta directamente la salud de la persona, pero que también se refleja o se manifiesta al interior del grupo familiar, generando repercusiones o correlaciones con los procesos que éste vive.

En esta etapa, se puede observar como la reunión de equipo es un espacio en el que se transita desde un trabajo multidisciplinario cuya expresión es el trabajo a solas de cada profesional que trabaja con la persona o familia, hacia un estilo interdisciplinario donde tiene lugar interacción de los distintos profesionales que pertenecen al equipo. Klein (1990, p. 141) sostiene en este sentido que, "un equipo interdisciplinario con una mirada comprensiva probablemente tiene mayores posibilidades de obtener un sentido de la realidad objetiva del paciente", por lo que si todos aquellos profesionales que tienen injerencia con el caso presentado participan y aportan a la descripción inicial, la intervención que se podrá planear hacia el fin de las conversaciones podría tener un impacto más certero.

\section{Análisis}

En esta etapa, uno o más miembros del equipo comienzan a emitir apreciaciones, opiniones y preguntas acerca de la historia relatada, se identifican reflexiones acerca de los procesos que está viviendo la familia (de normas, roles, límites, comunicación, etc.), en torno a por qué podría estar sucediendo tal o cual evento ahora, para luego generar incipientes aproximaciones hacia las posibles acciones y decisiones a tomar y las implicancias que puedan arrastrar.

También se aprecia cómo los expositores son interpelados por sus compañeros de equipo, buscando saber cuáles son sus propias inquietudes, exis-

4 Instrumento de registro gráfico de un árbol genealógico y que facilita un análisis intergeneracional de las familias, desde una perspectiva biopsicosocial. 
tiendo una constante retroalimentación respecto del trabajo ya experimentado y otorgando luces para comenzar a hilar lo que vendrá. Se denotan esfuerzos en los profesionales de los equipos por desarrollar reflexiones a la luz de elementos conceptuales y teóricos que sirven para el análisis de los casos presentados.

"Lo que pasa es que ojo también, porque también tiene que ver un tema de, bueno, obviamente, ellos dos como adultos no han marcado pa' na' los límites con los niños, pero tampoco límites entre ellos, o sea, está bien va todos los días, almuerza y todo... pero no es tan siquiera de vez en cuando..."

\section{Médico familiar}

“... Como que yo extraje a partir de esta familia, dos conceptos que tienen que ver como de... de la psicología... en el fondo pero pa' poder compartir un poco no es la idea profundizar tanto, pero si leerlos y a ver con como lo podemos asociar el tema del mito familiar y ... que es un poco lo que, lo que uno puede captar en el tema del niño y hay dos cosas que vemos como a diario, que lo hemos hablado de los mitos familiares... en como se expresa en los niños..."

\section{Psicóloga}

En esta etapa de la conversación se puede observar con claridad la riqueza de la forma de trabajo interdisciplinaria, pues cada profesional posee el espacio para manifestar su opinión desde su perspectiva y a su vez, para ir integrando nuevos lenguajes que van nutriendo las disciplinas y aportando en la construcción de respuestas integrales a las diversas preguntas que surgen en la interacción de las reuniones.

Es así como los aportes y contribuciones de cada miembro del equipo pueden ser revisados, redefinidos y reestructurados teniendo en cuenta a los otros hasta lograr un todo significativo, una integración sistémica, que podría expresarse con un modelo ya existente o de invención propia (Martínez, 2003, p. 116).

"¿Has solicitado un poco de ella qué expectativas... se han aterrizado las expectativas? En forma explícita? O sea... hasta aquí podemos llegar, esto no podemos hacer?..."

Médico familiar
"A propósito un poco de cómo vamos a iniciar el trabajo con la familia, con lo que decía la doctora, de ordenar lo más armónico posible las intervenciones, no sé si hay acuerdo en el equipo de que la primera intervención sea la citación a los papás para hacer esta invitación como padres, como pareja parental, no como personas individuales separada... ¿Hay acuerdo en eso...?

Trabajadora social

En este ámbito, se han esbozado consideraciones incluso desde la propia experiencia de vida de los miembros del equipo, donde se evidencia que la participación de los miembros no es del todo teórica y fundamentada en la trayectoria o la disciplina, sino que también proviene de la experiencia práctica y de la vida cotidiana.

La etapa del análisis es el espacio donde se puede notar con claridad la interacción de las diversas ocupaciones de los equipos de salud, ya que es posible expresar opiniones, pensamientos, ideas y reflexiones acerca de la práctica cotidiana de éstos. Todo ello constituye también una oportunidad para integrar conocimientos, aprender de los otros, llenar vacíos conceptuales existentes entre las diversas disciplinas y mejorar aspectos que emergen de situaciones críticas y muchas veces no previstas del trabajo diario. Esto es un proceso de co construcción entre quienes participan, que si bien, no son todos $^{5}$, contribuyen a generar una fructuosa integración de perspectivas diversas.

El aporte y la interacción de los miembros de un equipo interdisciplinario se genera cuando cada uno se siente coautor de sus objetivos, actividades y resultados. Para que alcancen su eficiencia esperada, deben desarrollar patrones funcionales de comunicación interna, flexibilización para acomodarse a las circunstancias que les aparezcan durante el trabajo, deben ser reconocidos por su esfuerzo y además tener orgullo de pertenecer a dicha agrupación (Scholters, Joiner \& Streible, 1999; Seaburn, 1996; Zurro, Recasens \& Cardona, 1999c. En Coimbra, A. 2002).

\section{La toma de decisiones}

La tercera parte de los diálogos acerca de las familias, se ha denominado de esta forma, justamente porque el sentido de los análisis realizados en cada 
uno de los casos particulares se dirigió hacia un "qué vamos a hacer", interrogante que se busca esclarecer hacia el final de las conversaciones.

En este sentido, se observan las decisiones, quedando planteadas las ventajas, desventajas e implicancias de tomar tal o cual camino. Cabe señalar que esta etapa es un proceso que se construye a partir de la interacción de quienes participan interviniendo, por lo que comprender por qué se toma un rumbo requiere reconocer que a través de los aportes individuales se va formando una opinión grupal y una decisión de equipo. De este modo, no es posible identificar el acuerdo con la persona que lo enuncia, ya que éste es un producto de toda la conversación. De allí que Martínez (2003, p. 116) señala que en el modelo interdisciplinario "la autoría compartida es la norma".

En general, los diálogos de las reuniones de los equipos de salud estudiados, recogen de manera significativa el trabajo que hay detrás de las atenciones otorgadas por los profesionales, encontrándose reflexiones grupales acerca de cuál determinación es conveniente tomar para los diversos casos. Se puede captar así, el reconocimiento tácito de la diversidad existente entre la población atendida, lo cual permite construir una forma de trabajo flexible y abierto a la evaluación de los avances, retroalimentación, supervisión y posible cambio de rumbo de la intervención.

Por otra parte, es importante reconocer la ventaja que se obtiene a partir de la diversidad de disciplinas que participan en las reuniones de equipo, ya que a través de su interacción durante las conversaciones, se genera el espacio propicio para que éstas se complementen, aprendan unas de otras, incorporen nuevos conocimientos y formas de intervenir, que no practicaban por la distancia existente en la formación de una carrera a otra. Klein (1990, p. 142) señala al respecto que el trabajo en equipo podrá tender a desdibujar las fronteras profesionales, por lo que "la comunicación continua es esencial, especialmente cuando el aprendizaje recíproco empieza a producirse".

Claramente, el intercambio de opiniones y apreciaciones respecto de una situación particular, entre un profesional proveniente de una disciplina bíomédica y otro de las ciencias sociales, en diálogos abiertos en los cuales cada uno expone sus perspectivas influido por sus principios profesionales y personales, evoca un trabajo interdisciplinario. Así, se complementan e integran las distintas miradas, pasando a formar parte de un cúmulo de saberes comunes de cada equipo y que se relacionan con formas de intervenir, tales como el trabajo en duplas profesionales, la intervención en crisis, la entrevista con los padres, la evaluación de la familia, la evaluación de la funcionalidad del adulto mayor, entre muchas otras.

\section{Participación}

Se aprecia en las reuniones registradas la tendencia de las trabajadoras sociales, las psicólogas, los médicos y enfermeras a participar en la presentación de la mayoría de las familias, lo cual, si se profundiza el análisis, se puede relacionar con que son los propios profesionales de aquéllas ocupaciones quienes atienden directamente a las familias, con los que a través del diálogo, se crea un vínculo de confianza, permitiendo la creación de un espacio para conocer tanto las fortalezas como las debilidades o dificultades que éstas atraviesan.

\section{Equipos interdisciplinarios: desafíos para Trabajo Social}

Gabriel Gyarmati (1991) conceptualiza como situs al conjunto de ocupaciones complementarias sobre sí, organizadas en torno de una determinada necesidad social. En el caso de la salud, reconoce la existencia de una profesión directora - la medicina - y otras complementarias - Psicología, Enfermería, Trabajo Social, Nutrición, Odontología, etc. - lo cual concuerda con la estructura de los CESFAM Áncora UC.

En cuanto a las percepciones por parte de miembros del equipo acerca de la utilidad de las reuniones, registradas en entrevistas en profundidad realizadas a trabajadores de los equipos de salud ${ }^{6}$, se denota conformidad y satisfacción respecto a las reuniones, en relación a los contenidos y aportes recibidos a través de su participación.

"Me parece que son... un super aporte... pa'l equipo, pa' la gente, pa'l modelo... pa' mi... por que yo nunca había trabajado en el área de la salud... no en cosas administrativas... otras cosas, pero nunca en el área de la salud (...). Esa mirada integral que se le da a las personas... super".

Recepcionista

6 Una matrona, una recepcionista, una paramédico, dos asistentes dentales, dos trabajadoras sociales, dos médicos familiares. (se incluyen entre éstas las dos jefas de los equipos). 
“... Yo no me imagino un trabajo mío, sola... o sea... la reunión de equipo es el espacio para saber que uno pueda compartir que está haciendo, que uno pueda revisar y supervisar lo que está haciendo, que uno pueda nutrir lo que está haciendo, hacerse preguntas que no se ha hecho, poder ver si lo está haciendo bien, efectivamente, si está trabajando integralmente o de repente muy solo, o sea también eso sirve para darte cuenta que a veces, efectivamente se ha ido trabajando temas muy desde lo social y a lo mejor requería mayor apoyo de equipo, o sea, creo que es fundamental..."

\section{Trabajadora Social}

"Para recibir educación... también nos dan educación sobre lo que es familia, lo que es el sistema de salud familiar y eso (...) igual se celebran los cumpleaños... para también para compartir como equipo, como grupo de trabajo (...) es más para aprender (...)"

\section{Asistente Dental}

“(...) Yo creo que como conocer a... al resto del equipo, eso se da sobre todo en las reuniones (...) si tu conoces mas o menos cómo trabajan los otros, tienes una mejor idea de eso (...) que todos tengan la posibilidad de... dar su opinión (...) de conocer la información que está dando vueltas (...) pa' ir viendo que algunas dudas que tú tienes también le pasan a los otros (...) tienes la posibilidad de compartir esas dudas con alguien más porque también le ha pasado antes..."

\section{Médico familiar}

En consecuencia, el trabajo interdisciplinario puede contribuir a mitigar los elementos tautológicos que tienden a contener las ciencias, ya que éstas emplean conjuntos típicos de preguntas para analizar los fenómenos naturales y sociales que constituyen su campo de estudio, usando un determinado aparataje teórico y metodológico para contestarlas. Al integrar, en vez de simplemente agregar, otra disciplina a los estudios, a menudo lo que se obtiene no es una nueva respuesta a la pregunta original, sino más bien se opera un cambio en la formulación de la pregunta misma (Gyarmati, s.n., p. 9).

De acuerdo a Klein (1990, p. 183) habrían ciertas habilidades o capacidades que caracterizan a quienes trabajan interdisciplinariamente. Mirar las cosas desde diferentes perspectivas, además de la capacidad de diferenciar, comparar, contrastar, relacionar, clarificar, reconciliar y sintetizar, serían elementos constituyentes del trabajo interdisciplinario. Saber como aprender nuevas habilidades frente a nuevos escenarios o contextos, saber cómo adquirir los conocimientos básicos de lenguaje, conceptos e información, teniendo capacidad analítica frente a problemas, procesos o fenómenos dados, son otras habilidades interdisciplinarias.

Surgen reflexiones durante las propias reuniones en las que se invita explícitamente a realizar un trabajo interdisciplinario, frente a la recurrente participación activa de los médicos familiares y profesionales del área psicosocial. En este sentido, el llamado se dirige al resto de los miembros del equipo (dentistas, enfermeras, matronas, asistentes dentales, paramédicos, recepcionistas, etc.). Dicha invitación refleja una valoración por la discusión e integración de ópticas distintas, para la construcción de respuestas nuevas (biopsicosociales) en relación con el trabajo con familias que se realiza durante la reunión de equipo.

“... Yo quería hacer una... en relación a la... a la presentación de la familia, como también hacer un llamado al equipo completo como pa' presentar. Tal vez, podría cualquiera de nosotros, traer... no sé la matrona, dentista...la enfermera ... y eso no está sucediendo, como que suele pasar que... a veces no traen familias o a veces... no sé, como llamado de atención tal vez, como para... entrar en las otras visiones también..."

Trabajadora social

“(...) Yo creo que siempre uno se imagina, en el abordaje de familias cuando era un rollo psicosocial pero a veces uno puede plantear un problema de familia (...) que surgen de problemas biomédicos que a veces trasciende un poco más y que están mal manejados porque en el fondo necesitamos coordinarlos, (...) y el tema es que detrás de eso está de que hay descuido de uno mismo, de alimentación y que se yo, y ese tipo de cosas uno también las puede conversar acá y plantear un trabajo en conjunto, de qué forma podemos en conjunto poder coordinar la educación (...)"

Médico familiar

“...La invitación a que todos y todas estemos como sensibles con la mirada y que nos pongamos con el tema y que traigamos aquí familias pa'... para que a la vez todos participemos en el análisis o en la busca de... mejoras abordables, porque en el fondo, no solamente traerlas, sino que qué podemos aportar cada uno desde sus miradas, desde sus experticias, o sus lugares de acción..."

Psicóloga 
Por otra parte, de acuerdo a los relatos de las reuniones de equipo observadas así como las narraciones de las entrevistas semi estructuradas, se identificaron actividades externas a la reunión de equipo semanal, que forman parte de las diversas instancias de trabajo que se orientan a integrar disciplinas, de manera tal que éstas sean algo más que complementarias. Entre éstas se encuentran:

Jornada de Evaluación del CESFAM a la que fueron convocados todos los trabajadores, de todas las ocupaciones para generar reflexiones e ideas comunes en torno al desarrollo y cumplimiento de objetivos, expectativas, etc., de la institución, durante el primer año de funcionamiento.

Reuniones de Salud Mental en la cual se integran a participar profesionales de diversas ocupaciones, con el fin de crear una herramienta para la colaboración en casos puntuales que se relacionen con la salud mental de los usuarios.

Capacitaciones realizadas por los mismos miembros de los equipos de salud, tanto a sus propios compañeros, como a la comunidad, las cuales se llevan a cabo, integrando a diversos miembros de los equipos. Las capacitaciones que se dirigen hacia los propios equipos de salud, ayudan a generar el espacio para que exista la integración de los aprendizajes entre las diversas ocupaciones. Esto, a su vez, es valorado por los miembros del equipo, como una de las actividades que se han realizado en las reuniones.

En torno a la reflexión acerca del trabajo interdisciplinario, Gyarmati señala que existen al menos dos formas de organización de los situs, de las cuales la salud no se escapa: el principio de exclusión hacia abajo y el principio de exclusión hacia arriba. El primero se caracteriza por que cada ocupación trata de incluir la mayor cantidad posible de actividades dentro de su esfera, dejando sólo las que a ella no le interesan para las ocupaciones de un nivel jerárquico inferior, lo cual permite delimitar los conocimientos que se conocen oficialmente a las diversas ocupaciones (Gyarmati, 1991).

De esta forma, el autor propone organizar los situs de acuerdo al segundo principio, debiendo preguntarse, qué tipo y volumen de funciones, actualmente desempeñadas por médicos, podrían desempeñar las enfermeras, tal vez, con un poco más de preparación. Con esta propuesta se busca aumentar los conocimientos de las ocupaciones intermedias para ampliar el volumen de responsabilidades que pueden asumir y no disminuir el nivel de preparación de los especialistas de alto nivel (Gyarmati, 1991).
Situación similar puede idearse en aquellas actividades comunes en la interacción entre médicos y profesiones no directoras que participan en la atención primaria de la salud como Psicología y Trabajo Social, donde es posible interactuar conjuntamente a través de la puesta en común de elementos que requieren intervenciones que no necesariamente requieren de la "ultima palabra del médico" para tener resultados positivos.

Un ejemplo de lo anterior es la experiencia relatada por Tobeña (2006, p. 17) a la luz de una investigación que aborda las relaciones de poder en un equipo interdisciplinario de salud, en donde las trabajadoras sociales desarrollaron una labor especial, complementaria y valorada por el resto del equipo, y en especial por los médicos, que cumplió un rol contextualizador, intentando construir la trama social en la que se inscriben las problemáticas que los usuarios plantean en las consultas, y asumieron la responsabilidad de ser guardianes del cumplimiento de la interdisciplinar.

Un hito que se considera importante destacar a modo de ejemplo en este ámbito, son las Visitas en terreno enunciadas por dos miembros de un equipo (asistente dental y paramédico) durante las entrevistas, lo cual denota una innovación en la forma de trabajar de acuerdo la distribución de las responsabilidades asignadas a los diversos trabajadores y el tipo de interacción que se establece entre los miembros del equipo y los usuarios. Esto, especialmente porque ambas señalan haber ido a terreno con la trabajadora social evidenciando la participación y responsabilidad que conservan ellas en el trabajo del equipo y por consiguiente la capacidad de la profesional para integrar y validar el trabajo de los miembros de su equipo.

"Si salí (a terreno) una vez (...) a ver un paciente... con la señorita Trabajadora Social, pero era una... en un terreno netamente de enfermería, o sea... no era como... más que eso... Tenía que ir ella con la enfermera ese día, pero la enfermera no estaba y me pidió que la acompañara... Pero fue entretenido si (...)"

Paramédico

"(...) Y eso es bueno también porque lo hacen participar... La jefa de equipo me ha llevado a terreno (...)"

Asistente dental

Finalmente, a través de los registros destaca el trabajo orientado a Contactos con vínculos de la red siendo un tema de conversación respecto de potenciales derivaciones a otras instituciones públicas 
o privadas, que permitan la realización de intervenciones integradas, en las que se entregue de la manera más completa la atención a los usuarios.

Cabe mencionar que los dos últimos ejemplos de trabajo interdisciplinario -visitas en terreno y contactos con vínculos de la red- se observan en los registros correspondientes al equipo cuya jefa es trabajadora social, quien en la mayoría de los casos marca la pauta, abre el tema y comenta instancias para derivar personas con el fin de optimizar la calidad de la atención. Cazzaniaga (2007, p. 107), señala respecto del segundo ejemplo que uno de los desafíos para el Trabajo Social en aquellos espacios institucionales donde no se cuenta con otros profesionales, es construir el equipo, trabajando interinstitucionalmente. En la práctica del día a día, es común realizar articulaciones con profesionales de diferentes instituciones u organizaciones, el desafío sería trascender la articulación, hacia una construcción interdisciplinaria.

En todas las actividades recién expuestas, se puede apreciar cómo a través de acciones como el lenguaje, intercambio de conocimientos, atención a los usuarios, reuniones de equipo, entre otros, los profesionales y no profesionales de los equipos ponen en común aspectos de su práctica cotidiana, los conocimientos que utilizan para ésta y los aprendizajes que han obtenido.

Lo que sucede al interior de los equipos de salud del CESFAM estudiado podría considerarse como un incipiente desarrollo de trabajo interdisciplinario, pues paulatinamente se han ido creando espacios en los cuales las diversas ocupaciones adquieren la capacidad para construir en conjunto, nuevas preguntas que contengan la óptica de más de una sola disciplina, lo cual otorga la posibilidad de analizar más a fondo las situaciones. Sin embargo, aún queda camino por recorrer, pues la interdisciplina no se produce con solo agrupar a las distintas profesiones en una reunión, sino que implica avanzar en la integración mutua de las disciplinas, permitiendo que todas participen en el proceso de construcción de nuevas interrogantes a la luz de las intervenciones que se realizan en equipos de salud.

La jerarquía y distribución de responsabilidades entre las diversas ocupaciones, adquiere un sentido especial al momento de analizar la interacción de éstas al interior de un equipo interdisciplinario, sin embargo, cabe señalar que cada uno de los equipos de salud observados es liderado por un profesional, que se ha designado por la dirección del CESFAM, siendo en este caso una Médico Familiar y una Trabajadora Social.
Esto conlleva diversas implicancias que se vinculan con el enfoque desde el cual cada jefa de equipo construye su liderazgo que se expresa en la información que entrega, en los contenidos de las reuniones, en la interacción grupal, en la construcción de objetivos y en los acuerdos tomados en cada reunión.

Respecto del liderazgo en los equipos interdisciplinarios de salud, Klein (1990, p. 143) plantea que el status profesional puede influir en la elección del líder, mostrando una discusión entre varios autores que sostienen posturas distintas en relación a la conducción de este tipo de equipos, donde algunos manifiestan su preferencia por los médicos mientras que otros recomiendan esta tarea para profesionales no médicos. Frente a ello, la autora resalta la importancia de diferenciar el rol de coordinador y de médico, pues si bien éste posee gran responsabilidad en las decisiones que se toman con determinados casos, la coordinación es una tarea distinta.

Tobeña agrega que las universidades tendrían un grado de responsabilidad en este tema, y refiere que "la construcción de la figura del médico que realizan las instituciones formadoras de médicos, imprime a fuego en sus estudiantes una imagen desproporcionada de la supremacía de este actor ante cualquier proceso terapéutico" (2006, p. 11).

Al respecto, Klein $(1990,157)$ describe experiencias de interdisciplinariedad en las universidades, donde se ha roto la dicotomía entre educación general y especializada, avanzando hacia una especialización de una disciplina que incluye el trabajo común con otras disciplinas.

De acuerdo a Gyarmati (1970), en un equipo interdisciplinario cada profesión va adquiriendo poder sobre las interpretaciones y definiciones de la realidad desplazando las definiciones emanadas por otros sectores. En concordancia con ello, cabe mencionar que el equipo que dirige al CESFAM, llamado Consejo Técnico, está compuesto por miembros de distintas disciplinas, cuyos integrantes son los jefes de cada equipo (médico familiar, trabajador social, jefe administrativo y enfermera universitaria), el coordinador de docencia (médico familiar), un coordinador de temas comunitarios (psicólogo) y el Director (médico familiar).

La inclusión de un Trabajador Social tanto en el equipo directivo como en los equipos asistenciales de salud, convoca de inmediato a destacar por una parte, aptitudes personales de quien es escogido para ocupar tal responsabilidad y por otra, reconocer aquellas competencias que tiene o debiera tener el profesional. Sin duda la importancia de la disciplina en los equipos de salud queda puesta en 
evidencia en los relatos mostrados en las páginas precedentes, donde se destacan algunas iniciativas interdisciplinarias protagonizadas por trabajadoras sociales.

Ahora bien, ¿son éstas competencias personales o profesionales?, claramente este estudio no pretendía dar cuenta de dicha interrogante, sino de la actuación en conjunto de los equipos, mas vale la pena pensar en el desafío de aportar una visión crítica que desde la profunda reflexión sobre el contexto social contribuya a desnaturalizar y desestigmatizar, generando capacidad reflexiva y dialogante frente a los procesos relacionados con la salud de las comunidades en un contexto de trabajo donde la tendencia es estandarizar y categorizar, prescribir e indicar soluciones.

En relación al éxito de los equipos interdisciplinarios y las habilidades o destrezas fundamentales para lograrlo, Klein (2006, p. 150) menciona que los profesionales deben ser capaces de adquirir, evaluar y utilizar la información adquirida desde variadas fuentes, a su vez, debieran ser capaces de juzgar cuales disciplinas son apropiadas para un problema particular y de colaborar con sus compañeros de equipo, además de derivar y ofrecer espacios de educación en caso de ameritarlo.

Trabajo Social, al ser una profesión que aporta una visión más bien alternativa a la tradicional biomédica, adquiere la oportunidad de ser escuchado y valorado por las profesiones de dicha tendencia. Por ello, aumentan las posibilidades de generar una transformación acorde al modelo de salud familiar desde dentro de los equipos de salud, que son quienes tienen en sus manos el poder de dar cabida y emprender en su accionar este nuevo enfoque propuesto.

Se multiplican entonces las alternativas de contribución de Trabajo Social a la luz de intervenciones interdisciplinarias, que no solo trascienden las parcialidades de estilos positivistas, sino que se abren posibilidades para abordar las demandas en su complejidad, permitiendo tanto la contención grupal de los profesionales como la elaboración de alternativas políticas de conjunto (Cazzaniga, 2007, p. 108).

\section{Formación interdisciplinaria en Trabajo Social: fortalecerla desde la práctica profesional}

Existen experiencias innovadoras de interdisciplinariedad en la formación de profesionales, a partir de las cuales surge el desafío para que en los centros de educación se implementen a partir de los curri- culums de las carreras, mallas integradas, cursos que incluyan estudiantes de distintas carreras, campos de práctica en donde los estudiantes de diversas carreras puedan aprender en conjunto su oficio trabajando en equipo (Klein, 1990).

Se abre el espacio entonces para discutir acerca de cómo se están gestando desde la formación de la profesión de Trabajo Social las estrategias para enfrentar el desafío de participar en equipos interdisciplinarios. Particularmente, desde el sector salud, se observa la tendencia a incluir cada vez con mayor intensidad la intervención social como parte importante de las acciones de promoción y participación, lugar que ocupan sin duda como profesionales expertos los trabajadores sociales. El modelo de salud familiar es parte de estas acciones que van dando relevancia a una profesión que en sus orígenes cumplió tareas de tendencia higienista con fines de sanidad pública, dado el contexto y las demandas de la época y que poco a poco ha ido resignificándose hasta alcanzar un rol protagónico en relación a la capacidad de gestionar redes, intervenir con familias y coordinar intervenciones interdisciplinarias, trabajando codo a codo con médicos, enfermeras, nutricionistas, psicólogos, en el modelo de salud familiar.

Claramente no es el único sector de las políticas sociales en el que Trabajo Social interviene con un rol paulatinamente más importante, ya en justicia, con la reforma procesal penal o el trabajo con población infanto juvenil, los trabajadores sociales están siendo requeridos en carácter de expertos que poseen la formación o al menos debieran tenerla, para intervenir socialmente en ámbitos específicos. No es casual la discusión acerca de la ubicación de la profesión en el lugar del medio, fortaleciendo la concepción de una labor que interviene entre fenómenos y contextos sociales distintos y que muchas veces actúa como nexo entre los sujetos de intervención y determinados contextos sociales, institucionales $\mathrm{u}$ otros. De acuerdo con ello, el trabajador social lleva a cabo su accionar justamente en la interacción del sujeto con su entorno, el cual se constituiría por el conjunto de personas y sistemas significativos para el sujeto, potenciales recursos de su red de apoyo (Latorre y Valdivieso, 1997, p. 32).

Consecuentemente, en vivienda, educación y cualquier otro sector de las políticas sociales, Trabajo Social aparece como profesión interviniente, con competencias esperables para desempeñarse en equipos de trabajo atingentes a cada uno de los requerimientos específicos del área donde con seguridad, el desafío consiste en generar políticas, 
programas e intervenciones sociales a la luz de discusiones al interior de equipos multi o interdisciplinarios.

Sin embargo, ¿estamos preparados para ello?, ¿están nuestras escuelas de Trabajo Social preparando profesionales con las competencias para afrontar el desafío de trabajar con otros y distintos?

Al menos la historia de la formación en Trabajo Social revela la inclusión de varios de estos ámbitos desde el origen, vale la pena memorar las materias que las escuelas Dr. Alejandro del Río (fundada en 1925) y Elvira Matte (fundada en 1929), incorporaron en la programación de los primeros años en que se dicta la carrera (Cuadro $N^{\circ} 1$ ). Es precisamente desde la configuración inicial de las escuelas de Trabajo Social -cuya gestación incluye la revisión y apoyo de profesionales provenientes de escuelas europeas en conjunto con profesionales médicos, diplomáticos, sacerdotes y otros- (Aylwin, et. al., 2004) en donde se aprecia la configuración interdisciplinar de la profesión de Trabajo Social, adquiriendo desde la formalidad del currículum la condición innata de interactuar con otros desde la complejidad de los fenómenos sociales.

Ahora bien, en la actualidad las mallas curriculares no distan en gran medida de aquella variedad de contenidos, sin embargo, es preciso hacer la diferenciación entre contenidos, en términos de saber de un tema -herramienta básica para trabajar en los distintos ámbitos potenciales donde se puede ejercer la profesión- y las competencias o habilidades a desarrollar para insertarse en equipos interdisciplinarios.
Llama la atención al menos en la experiencia de formación de la autora de este artículo -en el rol de estudiante y supervisora de prácticas profesionales de Trabajo Social- el constante encuentro con estudiantes de otras disciplinas en los distintos contextos laborales en que se desenvuelve la profesión, sin embargo, pese a la potencial articulación con otras disciplinas y experiencia de trabajo interdisciplinario desde la formación, esto no siempre tiene lugar pues no es parte de los objetivos de la práctica profesional el generar resultados interdisciplinarios.

Justamente en relación a lo anterior, Julie Thompson Klein (1990, p. 151) ha descrito cuatro modelos de educación en las ciencias de la salud, al referirse a la formación para un cuidado integral. El modelo tradicional (tradicional model) es el más común y predominantemente multidisciplinario, en éste los contenidos de los cursos son de una sola disciplina. El modelo de interés común (common-interest model) se enfoca en variados aspectos de la entrega de cuidados, incluyendo distintos temas relativos a la salud, tales como financiamiento, problemas éticos y estudios de la conducta. El modelo de presentación de casos (case presentation model) se aboca a analizar el caso particular de un paciente a la luz de elementos de estudio académico.

Finalmente, a través del modelo de equipo de salud (health team model), estudiantes de diversas disciplinas se hacen cargo conjuntamente de una tarea en algún equipo de investigación o de cuidados del paciente. Los equipos de investigación estudian un problema en particular, como

CUADRO No 1

MATERIAS INCLUIDAS EN EL CURRICULUM DE LAS PRIMERAS ESCUELAS DE TRABAJO SOCIAL EN CHILE

\begin{tabular}{|l|l|}
\hline Escuela Dr. Alejandro del Río & Escuela Elvira Matte \\
\hline Derecho y economía política & Religión \\
\hline Higiene y profilaxis & Psicología \\
\hline Pprotección a la infancia & Pedagogía \\
\hline Alimentación y dietética & Sociología y economía social \\
\hline Atención de enfermos y heridos & Asistencia Social \\
\hline Prácticas de secretaría & Legislación Social \\
\hline & Derecho de instrucción cívica \\
\hline & Anatonomía \\
\hline & Fisiología \\
\hline & Higiene pública y particular \\
\hline & Ética profesional \\
\hline
\end{tabular}

Fuente: Aylwin, N.; Fortes, A: Matus, T. La Reinvención de la Memoria. Indagación sobre el proceso de profesionalización del Trabajo Social Chileno 1925-1965, Ediciones Universidad Católica de Chile, Santiago, 2004, págs 66-70. 
por ejemplo, una encuesta de actitudes de una comunidad a través de métodos de investigación independientes o guiados, en cambio, los equipos de cuidados al paciente son responsables de un cuidado integral en el trabajo clínico. Por lo general, estos estudiantes están preocupados de la definición de su rol, la comunicación interdisciplinaria, el cuidado integral del paciente y el cuidado del paciente no institucionalizado.

Claramente, desarrollar el modelo de equipo de salud es un desafío que requiere una coordinación inter-escuelas, muchas veces entre diversas casas de estudios y por supuesto, con las instituciones que reciben practicantes. Es más, probablemente, guarda estrecha relación con las planificaciones estratégicas de las instituciones que incluyen o no la presencia, el trabajo y los resultados esperados de los estudiantes que concurren año tras año.

No obstante las numerosas barreras que pudieran oponerse a esta idea, el llamado es para los trabajadores sociales y profesionales de diversas ocupaciones que tengan la posibilidad de influir en sus instituciones.

¿Por qué no? Dada la experiencia mostrada en las páginas precedentes, donde queda en evidencia lo exitoso que puede ser el trabajo en equipos de profesionales que provienen de distintas disciplinas, si se establece el espacio y las condiciones para que todos participen y a través del consenso puedan generar intervenciones que trasciendan la utilidad de la intervención de una sola ocupación y perspectiva, ¿Por qué no incluir estas experiencias en las prácticas profesionales?.

La propuesta consiste en generar desde las propias instituciones que reciben practicantes las demandas de estudiantes de distintas disciplinas que se sumen a un plan común e interdisciplinario de intervención, sirviendo efectivamente a los requerimientos del contexto social que intervienen, a la planificación de la misma institución que recibe y supervisa estudiantes en práctica y a los objetivos de formación de sus escuelas. Es claro que la fuerza de un cambio en esta línea requiere voluntades institucionales, sin embargo puede provenir desde al menos dos de los actores involucrados en la toma de decisiones en relación a los alumnos en práctica:

- Profesionales que trabajan en la planificación de los programas de sus instituciones u organizaciones, en equipos interdisciplinarios y que reciben alumnos en práctica cada año.

- Escuelas de Trabajo Social y otras disciplinas que interactúan con la profesión (profesionales vin- culados a la supervisión de práctica profesional).

- Casas de estudios superiores, instituciones que pueden generar en sus proyectos educativos y programas curriculares la noción de formación interdisciplinaria.

Es esta propuesta un desafío que podría contribuir a la generación de competencias y habilidades de trabajo interdisciplinario para los trabajadores sociales y otros profesionales que compartan en un equipo que se proponga intervenir lo social.

\section{Conclusiones}

La reunión de los equipos estudiados es un lugar privilegiado para desarrollar un trabajo de calidad que se acerca a la noción de modelo de equipo de salud, en el cual el espacio para la reflexión y el análisis de la práctica se encuentra reservado. La experiencia que cada miembro del equipo ha puesto en común con sus compañeros de trabajo, ha sido un gran aporte ya que permite un enriquecimiento de las intervenciones llevadas a cabo, poniendo en evidencia que las reuniones de los equipos de salud estudiados, permiten la puesta en marcha del trabajo interdisciplinario para la integración del modelo de salud familiar, siendo especialmente la participación de todos y la disposición a aprender, elementos que le otorgan un valor agregado a diálogos y al aprendizaje de unos y otros.

En relación con el trabajo en equipo, se ha logrado entrever la valoración positiva por parte de los miembros de los equipos hacia las labores que cada cual desempeña y el trabajo en conjunto, demostrando, como antes se dijo, la importancia de las reuniones de equipo para las múltiples necesidades que surgen en los equipos, a partir del quehacer cotidiano y fortaleciendo la noción de proceso integrativo que caracteriza la interdisciplinariedad.

Con respecto a la interacción grupal de los equipos ha quedado registrada la percepción positiva de las relaciones humanas que se dan al interior de éstos. De esta forma, los espacios reservados para la reflexión y análisis de la práctica de los trabajadores, la participación de los más posibles en las discusiones, la adecuada y responsable capacitación y formación de éstos en relación a los temas que incorpora el trabajo del CESFAM, la satisfacción con el trabajo, el ambiente laboral, la forma en que se distribuyen las tareas y responsabilidades, son elementos que se identifican a lo largo de este estudio y que dan sentido al trabajo de lo equipos de salud, de acuerdo al modelo de salud familiar y en concordancia con la interdisciplinariedad. 
Finalmente y en relación con los desafíos que para Trabajo Social emergen a partir de los resultados del estudio y reflexión precedentes, cabe destacar las múltiples posibilidades y desafíos que ofrece la formación y el campo laboral para la carrera en la actualidad, siendo de gran interés el aporte que potencialmente está llamada a entregar en los diversos ámbitos en los que se desenvuelve.

En este sentido, es relevante el training ${ }^{7}$ que reciben los futuros trabajadores sociales respecto de sus competencias y habilidades para desarrollar su trabajo al integrarse en equipos interdisciplinarios. Si bien ya se encuentran incluidos - a juicio de la autora de este artículo- varios elementos para trabajar interdisciplinariamente, tales como una visión integradora de diversas perspectivas, relacionar y sintetizar, capacidad de aprender nuevas habilidades frente a nuevos escenarios o contextos, saber cómo adquirir los conocimientos básicos de lenguaje, conceptos e información, capacidad analítica y crítica frente a contextos diversos, la experiencia de aprendizaje e integración mutua, con otros profesionales durante la formación y práctica podría ser un gran valor agregado a las competencias.

La oportunidad radica en que la contribución de la profesión puede influir en el desarrollo de equipos que trasciendan la multidisciplina y avancen hacia un estilo de trabajo interdisciplinario, el cual implica síntesis e integración de los conocimientos, con el fin de favorecer el mejoramiento de la eficiencia y efectividad del uso de los recursos (tiempo, materiales y humanos) en los diversos contextos en los que se incorpora y además, realizar un auténtico aporte a la calidad de vida de los ciudadanos que usan los distintos servicios públicos que prestan las instituciones en las que los Trabajadores Sociales ejercen su profesión.

\section{Referencias}

AYLWIN, N., FORTES, A. \& MATUS, T. (2004). La Reinvención de la Memoria. Indagación sobre el proceso de profesionalización del Trabajo Social Chileno 1925-1965, Santiago: Pontificia Universidad Católica de Chile, Facultad de Ciencias Sociales, Escuela de Trabajo Social.

BARDIN, L. (1996). El Análisis de Contenido (2ºdición), Francia: Ediciones Akal.
CAZZANiGA, S. (2007). Hilos y Nudos. La formación, la intervención y lo político en el Trabajo Social. ( $1^{\circ}$ Edición), Buenos Aires: Editorial Espacio.

COIMBRA, A. (2002). Equipo Interdisciplinario en Salud Familiar Integral: evaluación de la satisfacción de los profesionales de salud del Programa de Familias en Riesgo. (Tesis presentada a la Escuela de Psicología de la Pontificia Universidad Católica de Chile, como requisito para optar al grado académico de Magíster en Psicología Clínica (s.n.).

GYARMATI, G. (1970). Las profesiones: dilemas del conocimiento y el poder. Santiago: Ediciones Universidad Católica de Chile.

GYARMATI, G. (1991). Salud y Enfermedad: Hacia un paradigma Biopsicosocial. Pontificia Universidad Católica de Chile, Facultad de Ciencias Sociales.

GYARMATI, G. (Documento sin antecedentes de publicación) El Concepto y método Interdisciplinarios. Pontificia Universidad Católica de Chile, Facultad de Ciencias Sociales, Instituto de Sociología (s.n.)

KLEIN, J. (1990). Interdisciplinarity: History, Theory \& Practice, Detroit: Wyne State University.

MARTÍNEZ, M. (2003, Julio). Transdisciplinariedad: Un enfoque para la complejidad del mundo actual, ConcienciActiva21, 1, 107-146. Recuperado el 20 de abril de 2009, de http://www.concienciactiva.org/ ?seccion $=$ ca2 1 \&pagina $=6$.

MATUS, T. (2005). Apuntes Sobre Intervención Social. Postítulo Intervención con familias en extrema pobreza. Universidad de Chile - FLACSO.

PÉREZ, G. (1994). Investigación cualitativa. Retos e interrogantes. II. Técnicas y análisis de datos. (3a Edición), Madrid - España: Editorial La Muralla S.A.

TOBEÑA, V. (2006, Febrero). Relaciones de poder al interior de un equipo interdisciplinario de salud, Kairos, Revista de temas sociales, 17, 1-22. Recuperado el 20 de abril de 2009, de http://www.revistakairos.org/k17-05.htm

VALDIVIESO, P., CAVIERES, H. (2004). Trabajo con Grupos. Postítulo Intervención con familias de extrema pobreza. Universidad de Chile - FLACSO.

\section{PÁGINAS WEB CONSULTADAS}

http://www.concienciactiva.org/? seccion=ca21\&pagina=6 http://www.who.int/peh-emf/research/agenda/es/index. html

http://www.revistakairos.org/k17-05.htm

www.Áncora.cl 